\title{
EFEITO DA PALMILHA DE REPROGRAMAÇÃO POSTURAL NA CAPACIDADE FUNCIONAL EM HIPERTENSOS
}

\section{EFFECT OF REPROGRAMMING POSTURAL INSOLE OF THE FUNCTIONAL CAPACITY IN HYPERTENSIVE}

\author{
Adonai Ferreira Dias*, Davi Moła de Jesus*, Murilo Cavalcante Negrão**, Taís Silva Nascimento*, \\ Vitor Pontes Soares**, Ana Lúcia Barbosa Goes***
}

\author{
Autor para correspondência: Adonai Ferreira Dias - adonaidias12.2@bahiana.edu.br \\ *Discente do curso de Fisioterapia na Escola Bahiana de Medicina e Saúde Pública (EBMSP) \\ **Bacharel em Fisioterapia pela Escola Bahiana de Medicina e Saúde Pública (EBMSP) \\ ***Mestre em Medicina e Saúde Humana pela Escola Bahiana de Medicina e Saúde Pública (EBMSP)
}

\section{R E S U M O}

\begin{abstract}
Introdução: Hipertensão Arterial Sistêmica (HAS) pode levar ao declínio da capacidade funcional (CF), promovendo inabilidade ou dificuldade em realizar tarefas do cotidiano. Existe tendência a relacionar excesso de estimulação do sistema nervoso simpático na manutenção da pressão arterial (PA) elevada. A Palmilha de Reprogramação Postural (PRP) ajusta a postura através de estímulos simpáticos em áreas semelhantes ao controle da PA. Objetivo: Verificar o efeito da PRP na capacidade funcional em hipertensos, e analisar o efeito da PRP nos parâmetros cardiopulmonares (PA, FC, FR e SpO2) e na percepção subjetiva do esforço. Métodos: Ensaio Clínico Randomizado, duplo-cego. O grupo intervenção $(G l)$ usou a PRP que contém o ressonador e o grupo controle (GC) usou palmilha sem $\circ$ artefato, por seis semanas. Realizadas duas avaliações (0-6 semanas). A avaliação da CF ocorreu através do teste de caminhada de seis minutos (TC6M). Para análise das variáveis distância percorrida, parâmetros cardiopulmonares e percepção subjetiva do esforço entre $G C$ e Gl utilizou-se o teste $T$ de Student e Mann Whitney. Para análise intragrupo, teste T de Student Pareado e Wilcoxon Sign Rank. Resultados: Após seis semanas de intervenção, não houve diferença nas distâncias percorridas entre os grupos, porém na análise intragrupo houve melhora de alguns parâmetros cardiopulmonares: PAS, PAD, SpO2, FC ( $<<0,05)$. Conclusão: A Palmilha de Reprogramação Postural não apresentou potencial para modificar a capacidade funcional de indivíduos hipertensos nessa amostra. Porém, na analise intragrupo demonstrou impacto nos seguintes parâmetros cardiopulmonares: PAS, PAD, SpO2 e FC, sugerindo otimização da atuação do Sistema Nervoso Autônomo.
\end{abstract}

Palavras-chave: Hipertensão. Teste de esforço. Atividade motora. 
Introduction: Systemic Arterial Hypertension (SAH) can lead to the decline of functional capacity (FC), promoting inability or difficulty in performing daily tasks. There is a tendency to link over-stimulation of the sympathetic nervous system in the maintenance of blood pressure (BP). The insole Postural Reprogramming (PRP) adjusts the position through friendly stimulus in areas similar to BP control. Objective: To determine the PRP effect on the functional capacity in hypertensive patients, and analyze the effect of PRP in cardiopulmonary parameters (BP, HR, RF and SpO2) and perceived exertion. Methods: Randomized Clinical Trial, double-blind. The intervention group (GI) used the PRP containing the resonator and the control group (CG) used insole without the artifact, for six weeks. Conducted two evaluations (0-6 weeks). The evaluation of the CF occurred through the six-minute walk test (6MWT). To analyze the variables distance, cardiopulmonary parameters and perceived exertion between GC and GI used the Student $t$ test and Mann Whitney. For intragroup analysis, Student's $t$ test and Paired Wilcoxon Sign Rank. Results: After six weeks of intervention, there was no difference in the distances between the groups, but the intra-group analysis was improvement in some cardiopulmonary parameters: SBP, DBP, SpO2, CF ( $p$ $<0.05)$. Conclusion: The insole Postural Reprogramming showed no potential to modify the functional capacity of hypertensive individuals in this sample. However, for analysis intragroup demonstrated impact in the following cardiopulmonary parameters: SBP, DBP, SpO2 and CF, suggesting optimization of the performance of the Autonomic Nervous System.

Keywords: Hypertension. Exercise Test. Motor activity. 


\section{INTRODUÇÃO}

A Hipertensão Arterial Sistêmica (HAS), condição clínica que promove a manutenção dos valores pressóricos em níveis elevados, tem caráter multifatorial $^{1,2}$. Em 2001, cerca de 7,6 milhões de mortes no mundo foram atribuídas à elevação da PA, a maioria em países de baixo desenvolvimento econômico e mais da metade entre 45-69 anos². No Brasil, a prevalência de HAS cresceu de $21,6 \%$ em 2006, para $42,4 \%$ em $2011^{3}$. Vale ressaltar a prevalência entre $22,3-43,9 \%$ na população em geral, com média de $32,5 \%$, com mais de $50 \%$ entre 60-69 anos e $75 \%$ acima de 70 anos $^{2}$.

O quadro hipertensivo irá promover inabilidade ou dificuldade na realização de determinadas tarefas que fazem parte do cotidiano, e, que normalmente são indispensáveis para vida saudável ${ }^{4,5}$. A capacidade funcional (CF) é definida como a habilidade do indivíduo de promover a realização de atividades físicas, de modo que consiga preservar as atividades mentais e consequentemente gerar integração social ${ }^{4}$. Apesar do estudo de Costa et al considerar que não houve diferença na distância percorrida observada e estimada em homens hipertensos ${ }^{6}$, estudos recentes tendem a demonstrar que a CF pode se apresentar com níveis reduzidos em indivíduos hipertensos ${ }^{7,8}$.

A CF pode ser mensurada através de diversos testes, dentre eles, 0 teste de caminhada de seis minutos (TC6M), também utilizado como preditor de morbimortalidade e na avaliação de intervenções terapêuticas comparativas $5,9,10$. O TC6M é considerado um teste submáximo que analisa de forma global todos os sistemas envolvidos durante o exercício, tais como musculoesquelético, cardiovascular e respiratório ${ }^{10,11}$. Porém, sabe-se que o sistema musculoesquelético apresenta impacto na função muscular e no declínio da capacidade funcional ${ }^{7,12,13}$.

As teorias que sustentam a manutenção da pressão arterial (PA) elevada tendem a considerar a ocorrência de excesso de estimulação do centro vasoconstrictor no córtex cerebral, por parte do sistema nervoso autônomo (SNA) $)^{11,13,14}$. Áreas do córtex cerebral que promovem ativação simpática da PA são semelhantes às áreas estimuladas pela
Palmilha de Reprogramação Postural (PRP), podendo ser destacado formação reticulada do bulbo e núcleos da base, porém, com desencadeamentos distintos. A PRP, muito utilizada para tratar alterações de postura, utiliza o SNA simpático como via de condução para ajustes do sistema tônico postural (STP). É constituída por um relevo com duas porções metálicas que se localizam na conexão do médio e retro pé situado em zona reflexa de estímulos somato-sensoriais, produzem frequência de vibração que, juntamente com as vibrações produzidas pelo corpo, irão sensibilizar receptores sensoriais, estimulando STP ${ }^{15}$.

Por existir semelhança nas áreas de ativação reflexa, acredita-se que a PRP possa demonstrar algum efeito na regulação da PA. Uma vez ocorrendo regulação dos níveis pressóricos devido ao uso da PRP, esperase a melhora da função muscular, otimizando a habilidade do indivíduo em desenvolver atividades físicas, e consequentemente, potencializar a CF. Apesar da PRP estar bem referenciada para correção da postura na literatura, não existe até o presente momento estudos que abordem o uso da palmilha em outras condições como a HAS e CF.

Portanto, o presente estudo tem como objetivo principal verificar $\circ$ efeito da Palmilha de Reprogramação Postural na capacidade funcional em indivíduos hipertensos e, secundariamente, analisar o efeito da PRP nos parâmetros cardiopulmonares (PA, FC, FR e SpO2) e na percepção subjetiva do esforço.

\section{MATERIAIS E MÉTODOS}

Ensaio Clínico Randomizado, duplo-cego (NCT02401516), cuja população acessível foi de hipertensos acompanhados no Ambulatório Docente Assistencial da Bahiana (ADAB) e no Complexo Comunitário Vida Plena (CCVP). Foram incluídos indivíduos com diagnóstico de Hipertensão Arterial Sistêmica (PAS $\geq 140 \mathrm{mmHg}$ e/ou $P A D \geq 90 \mathrm{mmHg})^{2}$, clinicamente estáveis, índice de massa corporal (IMC) 
até $34,9 \mathrm{Kg} / \mathrm{m} 2^{16}$, em uso regular de medicação anti-hipertensiva quando indicada, com faixa etária entre 30-60 anos e de ambos os sexos. Os critérios de exclusão abordados foram alterações musculoesqueléticas ou neuromusculares que limitem significativamente a caminhada, angina instável, história recente de arritmia cardíaca e infarto agudo do miocárdio.

Os indivíduos foram alocados em dois grupos por meio de sorteio: $\mathrm{Gl}$ - grupo intervenção e GC - grupo controle. Ambos os grupos foram submetidos ao uso de palmilhas. No GI, a PRP conteve o relevo com o ressonador que emite a corrente eletrogalvânica enquanto que a palmilha do GC não apresentava este artefato. Os participantes da pesquisa fizeram uso da palmilha no período de seis semanas, por pelo menos 12 horas por dia e com controle através do uso do mapa diário, elaborado pelos próprios pesquisadores.

O cálculo amostral foi realizado no WINPEPI (Published in Epidemiologic Perspectives \& Innovations) para comparação entre duas médias, com desvio padrão entre as medias de distância percorrida de 20 metros, com diferença das médias da distância percorrida esperada entre os grupos de $10 \%$, nível de significância de $5 \%$, poder do teste de $90 \%$, em hipótese bicaudal, totalizando 38 sujeitos, 19 sujeitos em cada grupo.

Foram recolhidos dados antropométricos, peso e estatura, através da balança manual (Welmy) e estadiômetro da própria balança, respectivamente. Em seguida foi aplicado questionário com informações pessoais, dados sóciodemográficos, hábitos de vida e saúde. Para aplicação do TC6M foram utilizados: corredor de $30 \mathrm{~m}$, cronômetro (celular iphone quatro), Tensiômetro digital (OMRON/HEM 433int), oxímetro de pulso (MD $300 \mathrm{Cl}$ ), trena (WISO T87), dois cones de trânsito, fita adesiva, uma cadeira e a escala de Borg modificada, para mensurar a percepção subjetiva do esforço.

TC6M foi aplicado segundo o guideline da American Thoracic Association ${ }^{10}$. $O$ teste foi realizado duas vezes, com $\circ$ intervalo de quinze minutos para garantir o efeito aprendizado. Foi utilizada a maior distância entre os dois testes. Após realização do TC6M, todos participantes receberam as respectivas palmilhas e orientações para utilização. Os mesmos foram acompanhados por contato telefônico na $3^{a}$ semana e na $6^{a}$ semana foram convidados a retornar para realização das avaliações iniciais (peso, estatura, PA, FC, SpO2, FR, PSE e TC6M.

As variáveis quantitativas idade (em anos), peso $(\mathrm{Kg})$, altura (centímetros), distância percorrida (metros), parâmetros hemodinâmicos (PA-mmHg, FCbpm, FR-ipm) e SpO2 (\%) foram expressas em média e desvio padrão. PSE foi expressa em mediana e intervalo interquartílico. As variáveis categóricas estado civil (Casado-União Estável/ Solteiro/Viúvo/ Separado-Divorciado), escolaridade (anos de estudo - até 4 anos, de 5-8 anos, de 9-11 anos e 12 ou mais anos), tabagismo (nunca fumou/ fumante/ ex-fumante), consumo de bebidas alcoólicas e sexo (dicotômicas), cor da pele (IBGE - Preta/ Parda/ Branca/ Amarela/ Indígena) foram descritas em valores absolutos e em porcentagem.

Foi realizada análise de intenção de tratar, em que todos os participantes do projeto foram acompanhados e tiveram seus dados coletados, mesmo os que desistiram do tratamento ou não usaram a palmilha de acordo com o orientado. Nesses casos, o resultado inicial das medidas foi repetido nas avaliações finais, após o período de intervenção.

$\neg$ efeito da PRP na CF foi identificado através da comparação das diferenças das médias de distância percorrida e dos parâmetros cardiopulmonares, entre os GI e GC, sendo utilizados os testes $T$ de Student e Mann Whitney. Para análise intragrupo, testes $T$ de Student Pareado e Wilcoxon Sign Rank, nível de significância de 5\%, em hipótese bicaudal. Os dados foram organizados no programa SPSS 14.0 para Windows. A pesquisa foi aprovada pelo Comitê de Ética e Pesquisa, CAAE 16952113.5 .0000 .5544 .

\section{RESULTADOS}

A amostra foi composta por 40 participantes, grupo intervenção $(n=19)$ e grupo controle $(n=21)$, as variáveis foram distribuídas de forma homogênea. 
Gl teve média de idade de $47,47 \pm 7,44$ e $49,81 \pm 7$ anos no GC, IMC de $28,14 \pm 4,50$ e $30,52 \pm 3,98 \mathrm{Kg} / \mathrm{m}^{2}$, circunferência de cintura $90,53 \pm 6,62$ e $95,92 \pm 5 \mathrm{~cm}$, respectivamente. No $\mathrm{Gl}, 47,4 \%$ eram casados, $57,9 \%$ tinham 12 ou mais anos de estudo, e 47,4\% referiram cor da pele parda. O GC apresentou as mesmas proporções, com exceção para cor da pele, em que 61,9\% referiram cor preta, tabela 1.

\begin{tabular}{|c|c|c|c|}
\hline \multirow[t]{2}{*}{ Variáveis } & Controle $(n=21)$ & Intervenção ( $n=19)$ & $P$ \\
\hline & Media $\pm \mathrm{DP}$ & Media $\pm \mathrm{DP}$ & \\
\hline Idade (anos) $\pi$ & $49,81 \pm 7,00$ & $47,47 \pm 7,44$ & 0,31 \\
\hline Altura $(\mathbf{c m}) \pi$ & $160,45 \pm 7,42$ & $160,37 \pm 9,01$ & 0,97 \\
\hline Peso $(\mathrm{Kg}) \pi$ & $78,36 \pm 9,63$ & $72,16 \pm 11,45$ & 0,07 \\
\hline \multirow[t]{2}{*}{ Diâmetro de Cîntura $(\mathbf{c m})^{\pi}$} & $95,92 \pm 5,00$ & $90,53 \pm 6,62$ & $0,00^{*}$ \\
\hline & $\mathbf{n}(\%)$ & $\mathbf{n}(\%)$ & \\
\hline Sexos & & & 0,72 \\
\hline Feminino & $15(71,4)$ & $15(78,9)$ & \\
\hline Estado Civil" & & & 0,69 \\
\hline Casado/união estável & $14(66,7)$ & $9(47,4)$ & \\
\hline Solteiro & $4(19,0)$ & $8(42,1)$ & \\
\hline Separado/divorciado & $2(9,5)$ & $1(5,3)$ & \\
\hline Viưvo & $1(4,8)$ & $1(5,3)$ & \\
\hline Anos de Estudo (anos)* & & & 0,83 \\
\hline Até 4 & $1(4,8)$ & $0(0,0)$ & \\
\hline $5-8$ & $2(9,5)$ & $3(15,8)$ & \\
\hline $9-11$ & $4(19,0)$ & $5(26,3)$ & \\
\hline 12 ou mais & $14(66,7)$ & $11(57,9)$ & \\
\hline Cor da Pele (IBGE)* & & & 0,11 \\
\hline Preta & $13(61,9)$ & $8(42,1)$ & \\
\hline Parda & $8(38,1)$ & $9(47,4)$ & \\
\hline Branca & $2(10,5)$ & $2(10,5)$ & \\
\hline
\end{tabular}

Em relação ao uso de medicamento anti-hipertensivo no $\mathrm{Gl} 15(78,9 \%)$ estavam em uso e 12 indivíduos $(63,2 \%)$ relataram que não praticavam atividade física, tendo em vista que 13 pessoas $(68,4 \%)$ nunca fumaram e $12(63,2 \%)$ não consomem bebida alcoólica, e no GC ocorreu a mesma predominância, $17(81,0 \%)$, $14(66,7), 18(85,7 \%)$ e $13(61,9 \%)$, respectivamente, tabela 2 . O Gl teve média na distância percorrida de

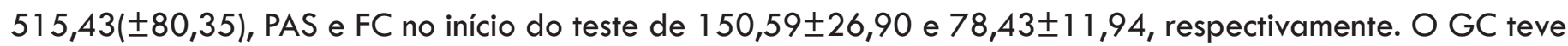

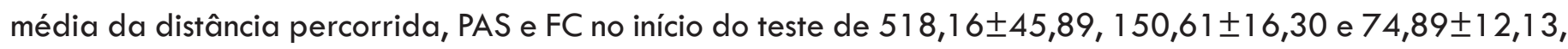
respectivamente, tabela 2 .

Tabela 2. Características da amostra quanto aos dados de hábitos de vida e parâmetros clínicos TC6.Salvador-Ba. 2016. (n=40)

\begin{tabular}{|c|c|c|c|}
\hline Variáveis & $\begin{array}{c}\text { Controle }(\mathbf{n}=21) \\
\mathbf{n}(\%)\end{array}$ & $\begin{array}{c}\text { Intervenção }(\mathbf{n}=19) \\
\mathbf{n}(\%)\end{array}$ & $\mathrm{P}$ \\
\hline \multicolumn{4}{|l|}{ Hábitos de Vida } \\
\hline Tabagismo" & & & 0,25 \\
\hline Nunca & $18(85,7)$ & $13(68,4)$ & \\
\hline Fumante & $0(0,0)$ & $1(5,3)$ & \\
\hline Ex-Fumante & $3(14,3)$ & $5(26,3)$ & \\
\hline Consumo de bebida alcoólica" & & & 0,93 \\
\hline Não & $13(61,9)$ & $12(63,2)$ & \\
\hline Medicamento anti-hipertensivo" & & & 1,00 \\
\hline Sim & $17(81,0)$ & $15(78,9)$ & \\
\hline Atividade física regular" & & & 0,81 \\
\hline Não & $14(66,7)$ & $12(63,2)$ & \\
\hline
\end{tabular}


Tabela 2. Características da amostra quanto aos dados de hábitos de vida e parâmetros clínicos TC6.Salvador-Ba. 2016. (n=40)

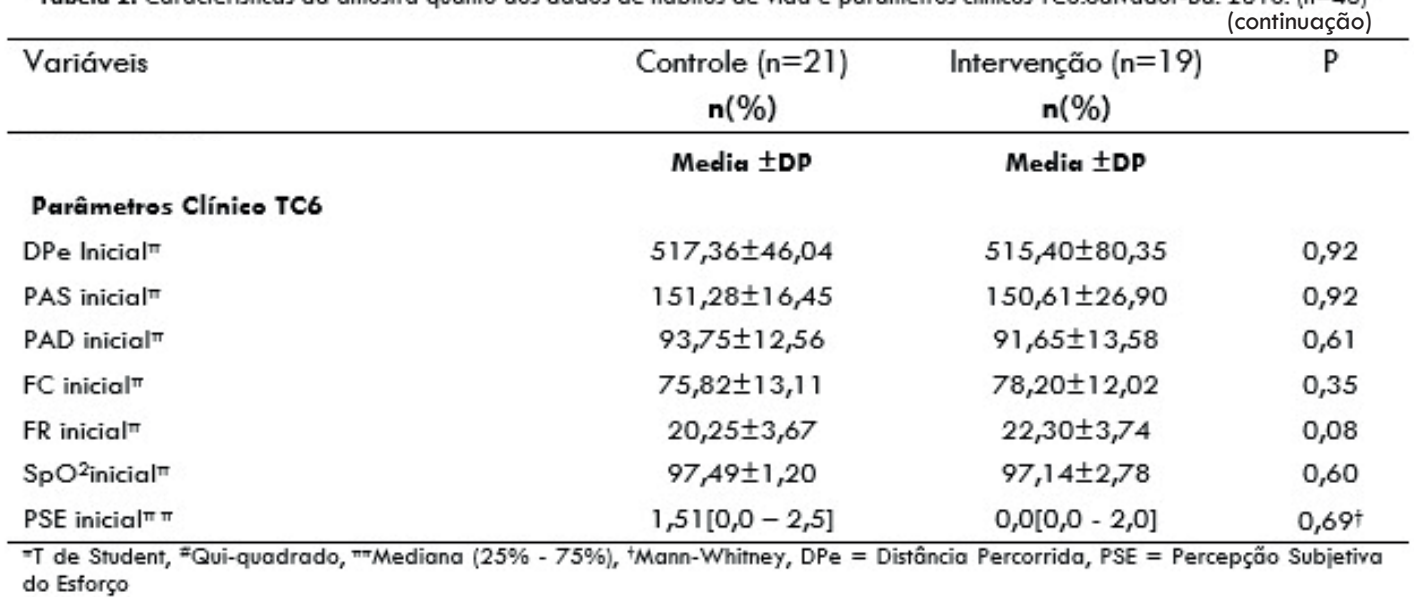

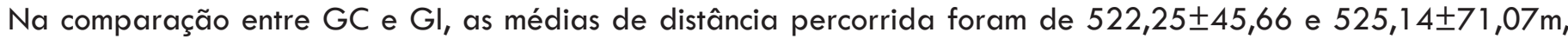
respectivamente, não apresentando significância estatística. Não houve diferença nas médias de PAS, PAD, FC, FR, $\mathrm{SpO} 2$ e percepção do esforço, tabela 3.

Tabela 3. Análise intergrupo das varióveis de distôncia percorrida e parâmetros cardiopulmonares após seis semanas. Salvador-Ba. 2016. $(n=40)$

\begin{tabular}{|c|c|c|c|}
\hline $\begin{array}{l}\text { Variáiveis } \\
\text { DPe e Parâmetros do TC6M }\end{array}$ & $\begin{array}{c}\text { Controle } \\
\text { Media } \pm D P\end{array}$ & $\begin{array}{l}\text { Intervenção } \\
\text { Media 士DP }\end{array}$ & $\mathbf{P}$ \\
\hline Distância Percorrida" & $522,25 \pm 45,66$ & $525,14 \pm 71,07$ & 0,87 \\
\hline Pressão Arterial Sistólica ${ }^{\pi}$ & $155,37 \pm 21,32$ & $150,15 \pm 22,95$ & 0,46 \\
\hline Pressão Arterial Diastólica" & $91,76 \pm 12,58$ & $91,89 \pm 10,31$ & 0,97 \\
\hline Frequência Cardíaca ${ }^{\pi}$ & $98,93 \pm 21,88$ & $101,13 \pm 6,93$ & 0,72 \\
\hline Frequência Respiratória" & $25,07 \pm 6,89$ & $25,50 \pm 4,61$ & 0,82 \\
\hline Saturação Periférica de Oxigênio" & $96,72 \pm 2,07$ & $97,27 \pm 0,97$ & 0,28 \\
\hline Percepção Subjetiva de Esforço" $\pi$ & $3,00[2,0-5,0]$ & $3,69[0,0-8,0]$ & $0,98^{\dagger}$ \\
\hline
\end{tabular}

TT de Student, ${ }^{* \pi}$ Mediana [25\% - 75\%], †Mann-Whitney, DPe=Distância Percorrida

$\mathrm{Na}$ análise antes e depois do grupo intervenção, foi observada melhora dos parâmetros cardiopulmonares PAS, PAD, FC e SpO2, com os seguintes resultados, antes da intervenção: 160,81 $\pm 23,41 \mathrm{mmHg}, 94,97 \pm 14,69 \mathrm{mmHg}$, $106,64 \pm 22,11$ bpm, 96,19 $22,65 \%$ e após a intervenção: $152,89 \pm 21,98 \mathrm{mmHg}, 91,82 \pm 11,41 \mathrm{mmHg}, 99,98 \pm 19,47$

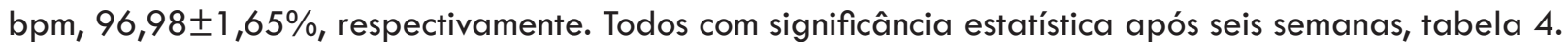

Tabela 4. Análise intragrupo das variáveis de distância percorrida e parâmetros cardiopulmonares após seis semanas. Salvador-Ba. 2016. $(n=40)$

\begin{tabular}{|c|c|c|c|}
\hline $\begin{array}{l}\text { Variáveis } \\
\text { DPe e Parâmełros do TC6M }\end{array}$ & $\begin{array}{c}\text { INICIAL } \\
\text { Medic } \pm D P\end{array}$ & $\begin{array}{c}\text { FINAL } \\
\text { Media } \pm D P\end{array}$ & $\mathbf{P}$ \\
\hline \multicolumn{4}{|l|}{ Distância Percorriela" } \\
\hline $\begin{array}{l}\text { Controle } \\
\text { Intervenção }\end{array}$ & $\begin{array}{l}517,36 \pm 46,04 \\
516,43 \pm 63,78\end{array}$ & $\begin{array}{l}522,25 \pm 45,66 \\
523,62 \pm 58,33\end{array}$ & $\begin{array}{l}0,54 \\
0,21\end{array}$ \\
\hline \multicolumn{4}{|l|}{ Pressão Arterial Sistólican } \\
\hline Controle & $158,42 \pm 19,89$ & $155,37 \pm 21,32$ & 0,07 \\
\hline Intervenção & $160,81 \pm 23,41$ & $152,89 \pm 21,98$ & $0,00^{*}$ \\
\hline \multicolumn{4}{|l|}{ Pressão Arterial Diastólica" } \\
\hline Controle & $95,24 \pm 16,23$ & $91,76 \pm 12,58$ & 0,10 \\
\hline Intervenção & $94,97 \pm 14,69$ & $91,82 \pm 11,41$ & $0,04^{*}$ \\
\hline \multicolumn{4}{|l|}{ Frequência Cardíaca ${ }^{\pi}$} \\
\hline Controle & $106,17 \pm 25,76$ & $98,93 \pm 21,88$ & 0,07 \\
\hline Intervenção & $106,64 \pm 22,11$ & $99,98 \pm 19,47$ & $0,00^{*}$ \\
\hline \multicolumn{4}{|l|}{ Frequêneia Respiratória" } \\
\hline Controle & $25,50 \pm 5,23$ & $25,07 \pm 6,89$ & 0,76 \\
\hline Intervenção & $26,41 \pm, 93$ & $25,27 \pm 5,85$ & 0,16 \\
\hline \multicolumn{4}{|l|}{ Seaturaçẽo Periférica de Oxigênio" } \\
\hline Controle & $95,96 \pm 2,99$ & $96,72 \pm 2,07$ & 0,23 \\
\hline Intervenção & $96,19 \pm 2,65$ & $96,98 \pm 1,65$ & $0,05^{*}$ \\
\hline \multicolumn{4}{|l|}{ Percepção Subjetiva de Esforço" $\pi$} \\
\hline Controle & $4,00[2,0-5,5]$ & $3,00[2,0-5,0]$ & $0,70 \dagger$ \\
\hline Intervenção & $3,00[1,0-5,0]$ & $3,69[0,0-8,0]$ & $0,70 \dagger$ \\
\hline
\end{tabular}




\section{DISCUSSÃO}

O presente estudo verificou que a PRP não apresentou alteração na distância percorrida no TC6M, ou seja, a intervenção não demonstrou repercussão significante na CF de indivíduos hipertensos. Porém, na análise do grupo intervenção após seis semanas obteve-se diferença em alguns parâmetros cardiopulmonares, como: Pressão Arterial Sistólica, Pressão Arterial Diastólica, Frequência Cardíaca e Saturação Periférica de Oxigênio.

Houve predominância de indivíduos sedentários na amostra, em ambos os grupos. Já se sabe que nessa população ocorre diminuição da distância percorrida no TC6M, com consequente declínio da $\mathrm{CF}^{5,17}$. Essa condição acontece devido ao desempenho inadequado dos sistemas musculoesquelético e cardiorrespiratório $^{18-21}$. Existe a possibilidade que a magnitude do efeito do sedentarismo na DP teve um impacto maior do que o efeito da PRP.

Nesse estudo, houve diminuição dos valores pressóricos, clinicamente relevantes, com $\circ$ uso da PRP, apesar dos mesmos não repercutirem na DP/ CF de indivíduos hipertensos. Algumas hipóteses podem ser sugeridas a partir desse resultado, como, por exemplo, a possibilidade dos parâmetros cardiovasculares não terem sido baixos o suficiente para gerar impacto na perfusão muscular, e na otimização da função muscular, o que, possivelmente, seria determinante para a melhora da distância percorrida ${ }^{22}$.

Outra possibilidade diz respeito à ativação contínua do SNA. Sabe-se que o SNA tem como objetivo principal promover a preservação das condições do equilíbrio fisiológico interno em detrimento aos estímulos ambientais, favorecendo a realização das atividades e demandas diárias. No entanto, em indivíduos hipertensos, já existe alteração da homeostase, uma vez que o SNA simpático se apresenta com excesso de atividade, como forma de manutenção do débito cardíaco (DC), o que promove, consequentemente aumento da resistência vascular periférica (RVP), diminuição da perfusão muscular e possível comprometimento de sua função ${ }^{22,23}$.

A intervenção proposta pelo presente estudo não demonstrou potencial para modificar a CF do indivíduo hipertenso, porém, vale ressaltar que os indivíduos do $\mathrm{Gl}$ apresentaram valores de DP maiores quando comparados com o GC. Uma possível justificativa para esse resultado pode ser demonstrada através da circunferência abdominal, onde o GC apresentou valores maiores do que o $\mathrm{Gl}$. Na literatura já é abordado que indivíduos do sexo feminino com a circunferência abdominal aumentada sofrem impacto na capacidade funciona ${ }^{24}$. Sabe-se que a deposição de gordura na região abdominal pode influenciar o dispêndio de energia, a função pulmonar e o consumo de oxigênio ${ }^{25}$. Além disso, alterações biomecânicas como diminuição da fase de balanço com consequente aumento da fase de apoio, predispõe a deambulação menor e a velocidade mais lenta ${ }^{26}$.

Diversos autores abordam a relação entre atividade nervosa simpática muscular (ASNM) com a Hipertensão, evidenciando o aumento da ASNM em repouso, com liberação de noradrenalina circulante, aumento do tono vascular e da RVP ${ }^{27-31}$. Essa condição pode ter favorecido a manutenção dos valores pressóricos acima do limiar de normalidade, promovendo condição desfavorável para função muscular, e assim, impossibilitando a melhora da CF. Qualquer desequilíbrio no SNA possibilitará o desenvolvimento de sistema compensatório no organismo humano e, de certa forma, a incapacidade de realizações de atividades cotidianas, ou seja, declínio da capacidade funcional ${ }^{23}$. Como o SNA regula todos os sistemas do corpo, inclusive o cardiorrespiratório e musculoesquelético, alterações simpáticas da PA e FC impactam a performance muscular, promovendo ambiente desfavorável para atuação funcional adequada.

Pode-se sugerir, ainda, que a manutenção de valores pressóricos elevados de forma sistêmica, impacte no tecido musculoesquelético, devido a redução da densidade vascular, o que levará a diminuição da perfusão muscular, com consequente falha na absorção de nutrientes e oxigênio pelos tecidos, comprometendo o desempenho muscular e favorecendo a queda da $D P^{13}$.

A diminuição da frequência cardíaca após seis semanas de intervenção pode ser explicada através dos centros reguladores. Alguns autores comentam que a melhora da capacidade física pode exercer 
significativas alterações nos mecanismos neurais de controle autonômico cardíaco, melhorando os parâmetros cardiopulmonares ${ }^{32-34}$. Essas alterações estão diretamente associadas ao incremento da atividade vagal e a possível redução da atividade simpática ${ }^{33}$.

Houve redução dos níveis pressóricos, porém os mesmos não alcançaram os valores de normalidade. No entanto, a diminuição desses valores podem ter impactado, em algum nível, na melhora da $\mathrm{SpO} 2$, pela possível diminuição da RVP e melhora na perfusão, mesmo esses parâmetros se mantendo elevados ${ }^{35}$.

A pressão arterial média (PAM), associada com o debito cardíaco são os principais determinantes da perfusão dos órgãos, portanto, a manutenção de nível adequado de PAM propicia, em geral, adequada perfusão orgânica ${ }^{35,36}$. A manutenção ideal dos níveis pressóricos estabelece relação com a otimização da perfusão e carreamento de oxigênio, impactando na $\mathrm{SpO} 2^{36}$. O resultado do estudo demonstrou esse panorama.

O presente estudo apresentou como limitação perda de participantes no decorrer da pesquisa, com significativa magnitude, acima de $20 \%$. Como se trata de estudo pioneiro, faz-se necessário mais estudos que embasem os achados, e que possam abordar de forma mais fidedigna os efeitos da HAS e da PRP na CF. Por ser trabalho original, muitas são as possibilidades de estudos na área, com grande impacto para comunidade cientifica, mas principalmente para a vida e o viver do indivíduo hipertenso.

\section{CONCLUSÃO}

A Palmilha de Reprogramação Postural não apresentou potencial para modificar a capacidade funcional de indivíduos hipertensos. Porém, na análise intragrupo demonstrou impacto em nível de SNA, influenciando alguns parâmetros cardiopulmonares (PAS, PAD, FC e SpO2), apresentando relevância clinica significativa, possibilitando a formação de novas estratégias terapêuticas para o controle dessas variáveis, e assim, atingindo o contexto social desses indivíduos.

\section{CONFLITO DE INTERESSE}

Os autores informam que não há qualquer conflito de interesse neste trabalho.

\section{REFERÊNCIAS}

1. Nobre F, Coelho EB, Lopes PC, Geleilete TJM. Hipertensão arterial sistêmica primária. Med. 2013;46(3):256-72. doi: 10.1 1606/issn.21767262.v46i3p256-272

2. VI Diretrizes Brasileiras de Hipertensão. Arq Bras Cardiol. 2010;95(1 supl.1):1-51.

3. Piccini RX, Facchini LA, Tomasi E, Siqueira FV, Silveria DS da, Thumé E et al. Promoção, prevenção e cuidado da hipertensão arterial no Brasil. Rev Saude Pública. 2012;46(3):543-50. doi: $10.1590 /$ S0034-89102012005000027

4. Rosa TEC, Benício MHD, Latorre MRDO, Ramos LR. Fatores determinantes da capacidade funcional entre idosos. Rev Saúde Pública. 2003;37(1):40-8. doi: 10.1590/S0034-89102003000100008

5. Fernandes PM, Pereira NH, Santos ACBC, Soares MESM. Teste de caminhada de seis minutos: avaliação da capacidade funcional de indivíduos sedentários. Rev Bras Cardiol. 201 2;25(3):185-91.

6. Costa HS, Martini AM de B, Quirino FC, Severino $G$, Novais LD, Lima MMO. Capacidade funcional em homens hipertensos pela distância caminhada e correlação com valores preditos. Fisioter Mov. 2009;22(4):557-64.

7. Soares VP, Dias AF, Jesus DM De, Nascimento TS. Correlação entre força muscular e capacidade funcional em hipertensos. Rev Pesqui em Fisioter. 
2016;6(1):6-15. doi: 10.17267/2238-2704rpf. v6il.796

8. Negrão MC, Dias AF, Goes AB. Capacidade funcional em indivíduos hipertensos no TC6': corte transversal. 2015.

9. Iwama AM, Andrade GN, Shima P, Tanni SE, Godoy I, Dourado VZ. The six-minute walk test and body weight-walk distance product in healthy Brazilian subjects. Brazilian J Med Biol Res. 2009;42(11):1080-5. doi: 10.1590/S0100$879 \times 2009005000032$

10. Crapo RO, Casaburi R, Coates AL, Enright PL, Maclntyre NR, McKay RT, et al. ATS statement: guidelines for the six-minute walk test. Am J Respir Crit Care Med. 2002;166(1):1 11-7. doi: 10.1164/ajrccm.166.1.at1102

11. Pires SR, Oliveira AC, Parreira VF, Britto RR. Teste de caminhada de seis minutos em diferentes faixas etárias e índices de massa corporal. Rev Bras Fisioter. 2007;1 1(2):1 47-51. doi: 10.1590/ S1413-35552007000200010

12. Irigoyen MC, Consolim-colombo FM, Krieger EM. Controle cardiovascular: regulação reflexa e papel do sistema nervoso simpático. Revista brasileira de hipertensao. $2001 ; 8(1): 55-62$.

13. Monteiro HL, Rolim LMC, Squinca DA, Silva FC, Ticianeli CCC, Amaral SL. Efetividade de um programa de exercícios no condicionamento físico, perfil metabólico e pressão arterial de pacientes hipertensos. Rev Bras Med do Esporte. 2007;13(2):107-12. doi: 10.1590/S151786922007000200008

14. Sevre K, Lefrandt JD, Nordby G, Os I, Mulder M, Gans RO et al. Autonomic function in hypertensive and normotensive subjects: the importance of gender. Hypertension. 2001;37(6):1351-6. doi: $10.1161 / 01$. HYP.37.6.1351

\section{Bricot B. Posturologia clínica. 2011 1. P. 264}

16. Associação Brasileira para o Estudo da Obesidade e da Síndrome Metabólica (Abeso). Diretrizes Brasileiras de Obesidade 2009-2010.
Associação Brasileira para o Estudo da Obesidade e da Síndrome Metabólica. 2009. P. 11-83

17. Steffen TM, Hacker TA, Mollinger L. Berg Balance Scale, Timed Up \& Go. 2002;82(2):1 2837.

18. Guyatt GH, Pugsley SO, Sullivan MJ, Thompson PJ, Berman L, Jones NL et al. Effect of encouragement on walking test performance. Thorax. 1984;39(1 1):818-22.

19. Lima SC, Caierão QM, Durigan JLQ, Schwarzenbeck A, Silva CA, Minamoto $V$ et al. Curto período de imobilização provoca alterações morfométricas e mecânicas no músculo de rato. Rev Bras Fisioter. 2007;1 1(4):297-302. doi: 10.1590/ S1413-35552007000400009

\section{Aquino CF, Viana SO, Fonseca ST.}

Comportamento biomecânico e resposta dos tecidos biológicos ao estresse e à imobilização. Fisioter em Mov. 2005; 18(2):35-43.

21. Santos TC, Travensolo CF. Comparação da força muscular respiratória entre idosos sedentários e ativos: estudo transversal. Rev Kairós Gerontol. $2011 ; 14(6): 107-21$

22. Laterza MC, De Matos LDNJ, Trombetta IC, Braga AMW, Roveda F, Alves MJ et al. Exercise training restores baroreflex sensitivity in nevertreated hypertensive patients. Hypertension. 2007;49(6):1298-306. doi: $10.1161 /$ HYPERTENSIONAHA.106.085548

23. Chaunchaiyakul R, Groeller H, Clarke JR, Taylor NA. The impact of aging and habitual physical activity on static respiratory work at rest and during exercise. Am J Physiol Lung Cell Mol Physiol. 2004;287(6):L1098-106. doi: 10.1152/ ajplung.00399.2003

\section{Campanha-Versiani L, Silveira ÉCBR,} Pimenta MC, Alvarenga SG, Parentoni $A N$ et al. Influência da circunferência abdominal sobre o desempenho funcional de idosas. Fisioter e Pesqui. 2010;17(4):327-31. doi: 10.1590/S180929502010000400008

25. Zamboni M, Mazzali G, Fantin F, Rossi A, Di 
Francesco V. Sarcopenic obesity: A new category of obesity in the elderly. Nutr Metab Cardiovasc Dis. 2008; 18(5):388-95. doi: 10.1016/i. numecd.2007.10.002

26. Wearing SC, Hennig EM, Byrne NM, Steele JR, Hills AP. The biomechanics of restricted movement in adult obesity. Obes Rev. 2006;7(1):13-24. doi: $10.1111 / \mathrm{i} .1467-789 x .2006 .00215 . x$

27. Roque JM a. Ariabilidade da. Fac Ciências do Desporto e Educ Física - Univ Coimbra. 2009;1-32

28. Tsioufis $C$, Kordalis A, Flessas D, Anastasopoulos I, Tsiachris D, Papademetriou V et al. Pathophysiology of resistant hypertension: the role of sympathetic nervous system.

Int J Hypertens. 2011 ; 201 1:642416. doi: $10.4061 / 2011 / 642416$

29. Bruno RM, Ghiadoni L, Seravalle G, Dell'oro R, Taddei S, Grassi G. Sympathetic regulation of vascular function in health and disease.

Front Physiol. 2012;3:284. doi: 10.3389/

fphys.2012.00284

30. Charkoudian N, Joyner MJ, Sokolnicki LA, Johnson CP, Eisenach JH, Dietz NM et al. Vascular adrenergic responsiveness is inversely related to tonic activity of sympathetic vasoconstrictor nerves in humans. J Physiol. 2006;572(P+ 3):821-7. doi: 10.1113 /iphysiol.2005.104075

31. Floras JS. Epinephrine and the genesis of hypertension. Hypertension. 1992;19(1):1-18. doi: 10.1161/01.HYP.19.1.1

32. Thompson PD, Crouse SF, Goodpaster B, Kelley $D$, Moyna N, Pescatello L. The acute versus the chronic response to exercise. Med Sci Sports Exerc. $2001 ; 33$ (Supl6):S438-45.

33. Perini $R$, Veicsteinas $A$. Heart rate variability and autonomic activity at rest and during exercise in various physiological conditions. Eur J Appl Physiol. 2003;90(3-4):317-25. doi: 10.1007/ s00421-003-0953-9

34. Goldberger JJ, Le FK, Lahiri M, Kannankeril PJ, $\mathrm{Ng} \mathrm{J,} \mathrm{Kadish} \mathrm{AH.} \mathrm{Assessment} \mathrm{of} \mathrm{parasympathetic}$ reactivation after exercise. Am J Physiol Heart Circ
Physiol. 2006;290(6):H2446-52. doi: 10.1152/ ajpheart.01118.2005

35. Seiler S, Haugen O, Kuffel E. Autonomic recovery after exercise in trained athletes: Intensity and duration effects. Med Sci Sports Exerc. 2007;39(8):1366-73. doi: 10.1249/ mss.0b013e318060f17d

36. Diniz GCLM, Zin WA, Botoni FA, Castro AV, Rodrigues-Machado MG. A influência de duas frações inspiradas de oxigênio no padrão respiratório de pacientes sob desmame ventilatório. Rev Bras Ter Intensiva. 2009;21 (3):292-8. doi: $10.1590 /$ S0103-507X2009000300009 